\title{
UV Photofunctionalization Effect on Bone Graft in Critical One-Wall Defect around Implant: A Pilot Study in Beagle Dogs
}

\author{
Min-Young Kim, ${ }^{1}$ Hyunmin Choi, ${ }^{1}$ Jae-Hoon Lee, ${ }^{1}$ Jee-Hwan Kim,, Han-Sung Jung, \\ Jae-Hong Kim, ${ }^{3}$ Young-Bum Park, ${ }^{1}$ and Hong-Seok Moon ${ }^{1}$ \\ ${ }^{1}$ Department of Prosthodontics, Oral Science Research Center, BK21 PLUS Project, Yonsei University College of Dentistry, \\ Seoul, Republic of Korea \\ ${ }^{2}$ Division in Anatomy and Developmental Biology, Department of Oral Biology, Oral Science Research Center, BK21 PLUS Project, \\ Yonsei University College of Dentistry, Seoul, Republic of Korea \\ ${ }^{3}$ Department of Dental Technology, School of Medical and Public Health, Kyungdong University, Wonju, \\ Gangwon-do, Republic of Korea
}

Correspondence should be addressed to Hong-Seok Moon; hsm5@yuhs.ac

Received 19 August 2016; Revised 3 November 2016; Accepted 1 December 2016

Academic Editor: Wei-Jen Chang

Copyright (C) 2016 Min-Young Kim et al. This is an open access article distributed under the Creative Commons Attribution License, which permits unrestricted use, distribution, and reproduction in any medium, provided the original work is properly cited.

The purpose of this study was to compare and evaluate, through histomorphometric and radiological analysis, the effects of UV photofunctionalization on an implant placed over a critical defect area with and without a bone graft. Four female beagle dogs were first divided into control and bone graft groups. Each group was then subdivided into UV-treated and UV-untreated groups. The mandibular premolars in each dog were extracted. 12 weeks after extraction, implants were placed according to the condition of each group. Four and 12 weeks after implantation on left and right mandible, the dogs were sacrificed. The specimens were prepared for histomorphometric and micro-computed tomographic analysis. In both 4-week and 12-week groups, UV-treated implant surfaces showed better osseointegration than SA implant surfaces. Also, with implant surfaces placed over the critical defect with bone graft, UV photofunctionalization increased bone-to-implant contact (BIC) and new bone formation at the initial stage (4 weeks). Based on the results of this study, it can be suggested that UV photofunctionalization on the surface of implants placed over large critical defects with bone graft aids initial osseointegration and osteogenesis.

\section{Introduction}

In modern implant dentistry, implant therapy is known as the most effective treatment for edentulous patient and is regarded as the first treatment of choice for missing teeth. The success of dental implants depends on how directly new bone formation occurs on titanium surface after implant placement [1]. The initial amounts of osseointegration and osteogenesis are crucial factors in the success rate of implant therapy $[2,3]$. In recent studies, Ogawa et al. researched the effects of UV photofunctionalization on titanium surface and concluded that conversion of implant surface from bioinert to bioactive yielded better osseointegration and osteogenesis on titanium surface [4-6].
The success of implant therapy also depends on bone condition of the surgical site during implant placement, as surgery is not always under ideal conditions. When there is insufficient bone support from the surrounding bones and surfaces are not fully covered with bones, this may lead to a failure of the surgery in the long term. In the case of a large bone defect area, a bone graft may be used along with an implant placement and the bone graft becomes a scaffold around the implant. It was reported that migration of osteoblast and osteoinductive materials to the implant surface increases the probability of implant success in the long term [7-9]. On the other hand, however, some reported that bone graft materials arrested new bone formation at the outset by blocking proliferation of osteoblast [10-12]. Nevertheless, it is 
generally accepted that once grafting materials are absorbed and eventually disappear, the space is filled with new bone so that the level of osseointegration and osteogenesis eventually increases $[13,14]$.

Since there already exists previous study regarding positive effects of UV photofunctionalization on implant surfaces under insufficient bone condition [5], the purpose of this study was to compare and evaluate the effects of UV photofunctionalization on an implant placed over a critical defect area that did not heal over the duration of the study with and without a bone graft through histomorphometric and radiological analysis.

\section{Materials and Methods}

2.1. Experimental Animals. In this study, four female beagle dogs of twelve months old (weighing approximately $10 \mathrm{~kg}$ ) were used. As a prestudy preparation, scaling and plaque control were performed for periodontal health. After treatment, they were fed with liquid foods to prevent masticatory trauma during healing. Animal selection, management, surgical protocol, and all experiments were reviewed and approved by the Animal Care and Use Committee, Yonsei Medical Center, Seoul, Korea (Approval number 2010-0362).

2.2. Experimental Implants. A total of 32 sandblasting with alumina and acid etching (SA) surface-treated internal type implants (Osstem implant system, TS II SA Fixture, Busan, Korea) being $3.5 \mathrm{~mm}$ in diameter and $8.5 \mathrm{~mm}$ in length were used in this study. All implants used in the experiment were manufactured simultaneously and stored in a sealed container, being kept minimally exposed to the air immediately before placement.

2.3. Ultraviolet Photofunctionalization. Photofunctionalization was performed by treating implants with UV light for 15 minutes using a photo device (TheraBeam Affiny, Ushio Inc., Tokyo, Japan) immediately before implantation according to manufacturer's recommendation [1, 4-6] (Figure 1). Details specifications of a photo device were as follows: input voltage (AC 100 to $240 \mathrm{~V} \pm 10 \%$ ), input current (2.2 A max), temperature $\left(15^{\circ} \mathrm{C}\right.$ to $\left.30^{\circ} \mathrm{C}\right)$, humidity $(20 \%$ to $70 \% \mathrm{RH})$, and altitude (below 2,000 m).

2.4. Graft Materials. Demineralized freeze dried bone allograft (DFDBA) (SureOss-D, Demineralized Cortical Bone Powder, Hans Biomed Corp., Seoul, Korea) $200 \sim 850 \mu \mathrm{m}$ in particle size was used for grafting at bone defect.

2.5. Experimental Groups Design. First, all implant placement sites were divided into bone graft groups and control groups, with each group then subdivided again into UV-treated group and UV untreated group. The UV untreated group with bone graft was set to Group 2 (BG only), the UV-treated group with bone graft was set to Group 4 (UV/BG), the UV-treated group without bone graft was set to Group 3 (UV only), and finally the UV untreated group without bone graft was set to Group 1 (control). Eight SA surface implants were used for Group 4, eight SA surface implants for Group 2, four SA surface implants for Group 3, and lastly twelve SA surface implants for Group 1. In this study, the sample sizes of Group 3 were reduced since the UV photofunctionalization effect has already been proven in many previous studies $[1,5,6,15,16]$. Implants were placed symmetrically to reduce differences in the sites by matching the initial states. Four implants were placed from posterior area of the 1st premolar in the right and left side of mandible (Figure 2). The distance between each implant was $10 \mathrm{~mm}$ and the experiment was carried out using a split-mouth design to minimize the interindividual variability from the estimates of the treatment effect [17]. The implants placed in the left side of mandible had a healing period of four weeks (4-week group) and the other implants placed in the right side of mandible had a healing period of twelve weeks (12-week group) (Figure 3).

2.6. Surgical Procedure. All of the surgical treatments were carried out under general anesthesia. Both mandibular premolars (from first to fourth premolar) were extracted atraumatically. 12 weeks after the extraction of four teeth (P1, P2, P3, and P4) on right side of mandible, sequential drilling for implant placement was performed. The distance between centers of implants was $10 \mathrm{~mm}$. A one-wall bony defect in cuboid shape was then formed for each group. Those defects were uniformly $3 \mathrm{~mm}$ in depth and $5 \mathrm{~mm}$ in width (buccolingual and mesiodistal). The center of implant was then placed on the edge of the cuboid (Figure 4). Photofunctionalization of implants was performed for 15 minutes using a photo device immediately before implantation. $0.25 \mathrm{cc}$ DFDBA was gently packed into each bone defect until it filled the entire cavity (Figure 5). One week after the surgical procedure, stitching out was done. The same processes were executed on left side of mandible eight weeks later. The beagle dogs were sacrificed after 4 weeks.

2.7. Fabrication of Histologic Specimens. After buffering of the tissue samples with neutral formalin fixation for two weeks, micro-CT was taken. Next, the samples were dehydrated in increasing grades of ethanol and subsequently infiltrated in Technovit 7200 resin (Heraeus Kulzer, Dormagen, Germany). Following the embedding in the acrylic resin, the blocks were polymerized and sectioned in the mesiodistal plane using a cutting-grinding unit (Exakt Exakt 300, Heraeus Kulzer, Norderstedt, Germany). The tissue samples were processed for ground sectioning according to methods described by Donath and Breuner and the sections were stained in $\mathrm{H} \& \mathrm{E}$ (hematoxylin and eosin) for light microscopic examination [18].

2.8. Micro-Computed Tomographic Analysis. A microcomputed tomography scanner (SkyScan 1076, SkyScan, Aartselaar, Belgium) was used to measure the percentage of the amount of new bone formation around implants. The new region of interest (ROI), the area most prone to be affected by the UV photofunctionalization and bone grafting, was set up in a rectangular parallelepiped $3.5 \mathrm{~mm}$ in width (mesiodistal) from the center of implant, $3.0 \mathrm{~mm}$ in length (buccolingual), and $3.0 \mathrm{~mm}$ in height. The amount of bone was measured in this area (Figure 6). 


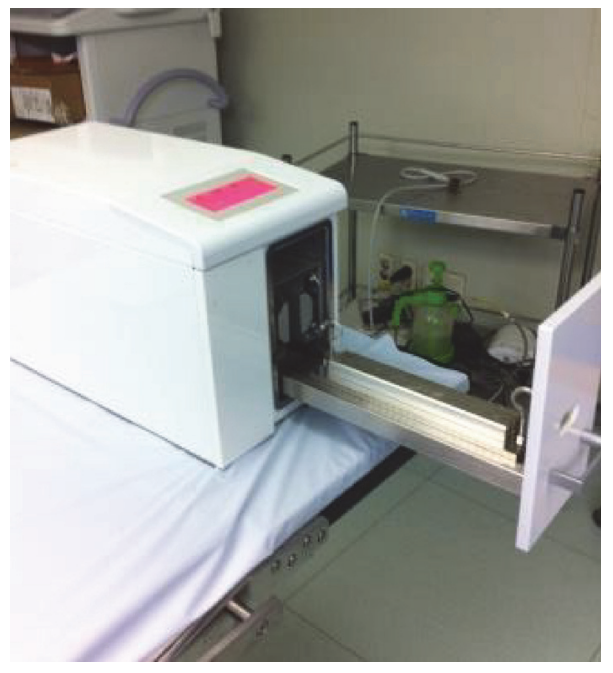

(a)

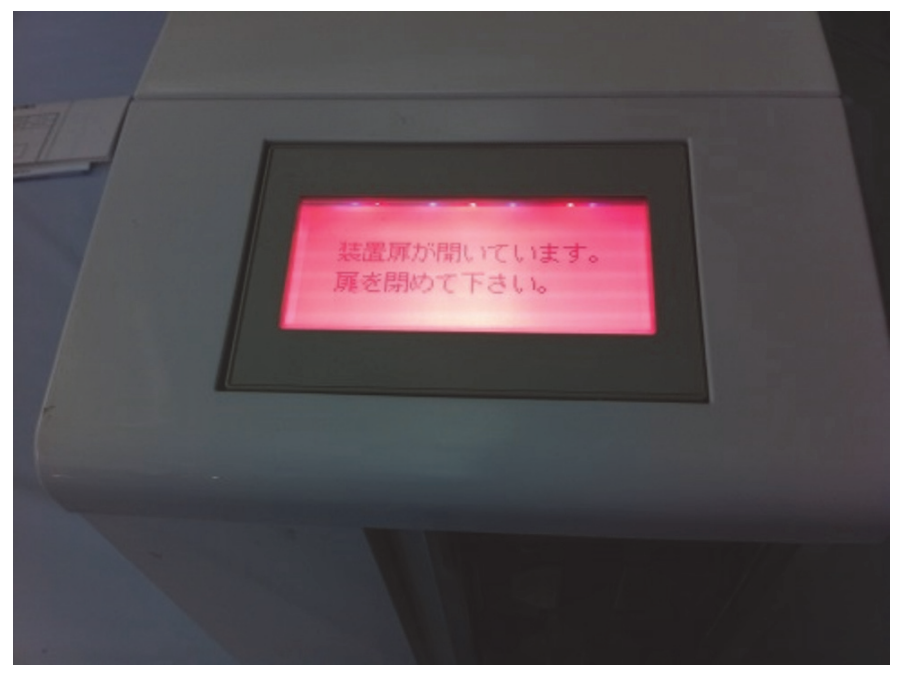

(b)

Figure 1: Photofunctionalization of implant fixture surface by ultraviolet light. (a) TheraBeam Affiny device (TheraBeam Affiny, Ushio, Tokyo, Japan). (b) Process of UV treatment on implant fixture during 15 minutes.

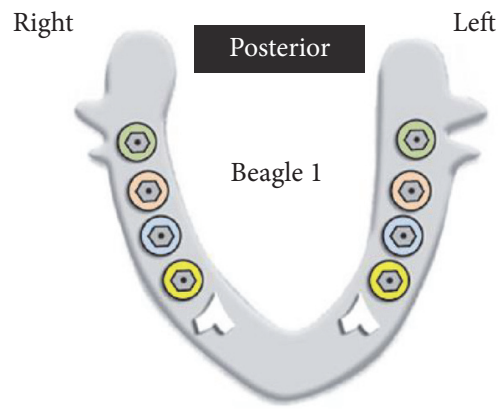

Anterior

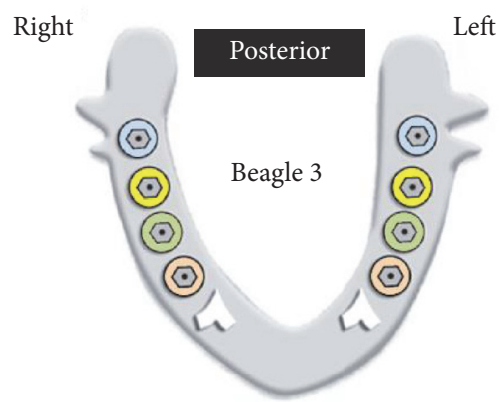

Anterior

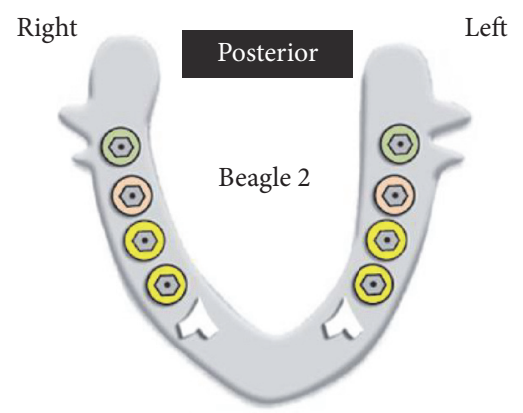

Anterior

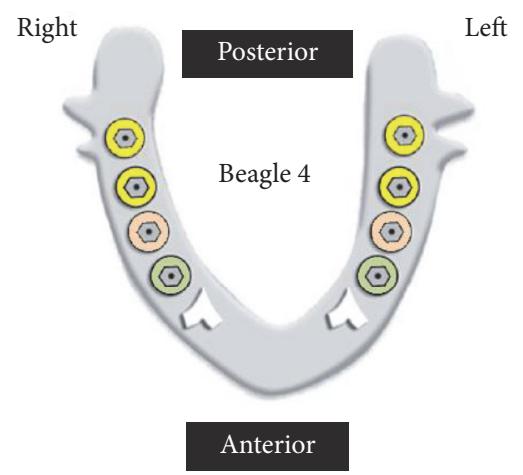
(-) Control
() UV
(๑) $\mathrm{BG}$
() $\mathrm{UV} / \mathrm{BG}$

FIgURE 2: Experimental schematic diagrams. 


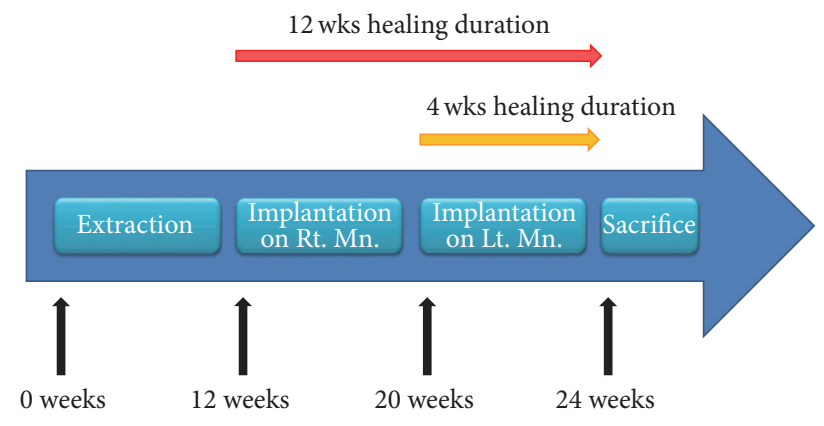

FIGURE 3: Diagram of experimental design protocol.

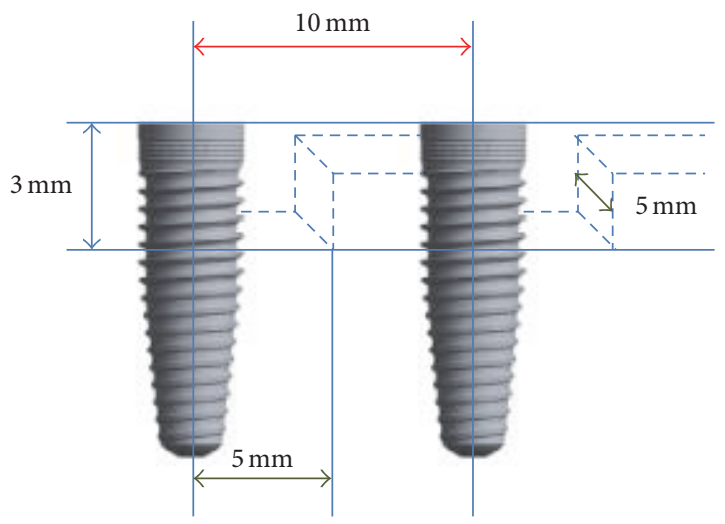

FIGURE 4: Schematic diagram of experimental design.

2.9. Histomorphometric Analysis. The stained histologic specimens were scanned and captured using optical microscopy at $12.5 \mathrm{x}$ and $50 \mathrm{x}$ magnification and then histomorphometrically measured using ImagePro Plus 4.5 (Media Cydernetrics, Silver Spring, Maryland, USA). At first, the actual zone of formed defect was set as ROI, the area most prone to be affected by the UV photofunctionalization and bone grafting, by sectionalizing the region on histologic specimens. Then, the ratio for bone-to-implant contact (BIC, $\%)$, new bone area formed in defect area (new bone area, \%), remaining graft material area in defect area (graft material area, \%), and resorption area in defect area (resorption area, $\%$ ) were calculated in ROI. BIC was measured at three consecutive threads in ROI. New bone area ratio and remaining graft material area ratio were calculated as a percentage of the area occupied by each one in ROI. Lastly, resorption area ratios were obtained as a percentage of the area in which bone filling did not occur in ROI (Figure 7).

2.10. Statistical Analysis. The ratios for BIC, new bone area, remaining graft material area, resorption area, and microCT value were compared to verify the difference between Groups 1 and 3. The same measurements were carried out for Groups 2 and 4. The Wilcoxon rank sum test was used as a nonparametric statistical method to evaluate the data due to the small sample size and large standard deviations. All calculations were performed using a specific statistical program (SPSS ver. 18.0, IBM Co., Somers, NY, USA), and the level of significance was set at $5 \%$.
TABLE 1: The mean of micro-CT value ratios at 4 and 12 weeks.

\begin{tabular}{lcc}
\hline \multirow{2}{*}{ Group } & \multicolumn{2}{c}{ Micro CT value ratio } \\
& 4 weeks & 12 weeks \\
\hline 1 & $12.33 \pm 4.52$ & $12.18 \pm 8.17$ \\
3 & $10.97 \pm 1.74$ & $7.62 \pm 6.95$ \\
4 & $14.37 \pm 13.55$ & $27.9 \pm 16.45$ \\
\hline
\end{tabular}

\section{Results}

3.1. Micro-Computed Tomographic Findings. For the comparison of cases without bone graft, in Groups 1 and 3, micro-CT bone volume ratio was measured in ROI (Figures 8 and 9). The mean value and standard deviation were then calculated (Table 1). Group 3 showed significantly increased micro-CT bone volume at 12 weeks compared to that of Group 1 ( $p<$ $0.05)$. However, for the comparison of cases with bone graft, in Groups 2 and 4, Group 4 displayed significantly increased micro-CT bone volume at 4 weeks compared to that of Group $2(p<0.05)$ (Figure 10).

3.2. Histomorphometric Findings. In Groups 1 and 3, BIC ratio (\%) was calculated. Although increased $\mathrm{BIC}$ ratio was identified in Group 3, there was no significant difference in $\mathrm{BIC}$ ratio between the two groups at either time point (4 weeks and 12 weeks). Also, no significant difference in BIC was identified between Groups 2 and 4 at either time point (Table 2 and Figure 11).

Regarding new bone area ratio (\%), a greater new bone area was identified in Group 3 than in Group 1. However, there was no significant difference in new bone area ratio between Groups 1 and 3. Also, in Groups 2 and 4, the similar results were identified but with no significant difference identified between two groups. Also, the remaining graft material area ratio (\%) was calculated and there was no significant difference between Group 2 and Group 4.

Although no significant difference was found, there was much reduced resorption area ratio (\%) observed in Group 3 compared to that in Group 1 at 12 weeks. Even when bone graft materials was used, decreased resorption area ratio was identified in Group 4 compared to that in Group 2. However, no statistically significant difference was found.

\section{Discussion}

The purpose of this study was to compare and evaluate, through histomorphometric and radiological analysis, the effects of UV photofunctionalization on an implant placed over a critical defect area with and without a bone graft. A large critical bone defect $5 \mathrm{~mm}$ in width was designed for the present study as the term "Critical Size Defect" (CSD) was defined in animal research as the size of a defect that will not heal over the duration of the study [19].

First, measurements for BIC ratio (\%) in ROI of each group were compared. The groups of implants placed over bone defect without graft yielded the similar BIC ratios at 4 weeks regardless of the presence of UV treatment. Conversely, 


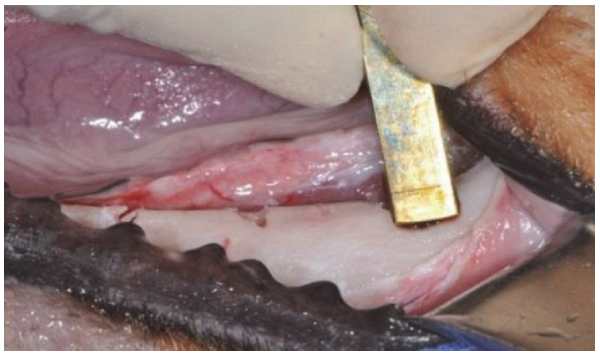

(a)

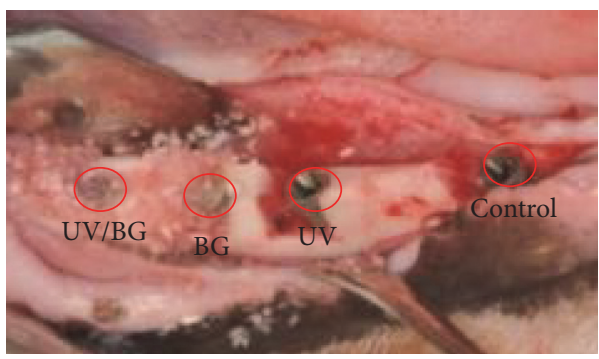

(c)

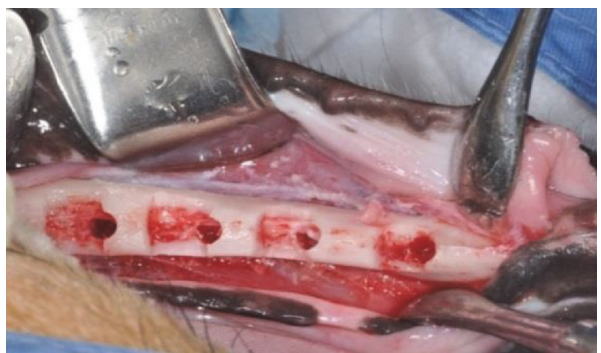

(b)

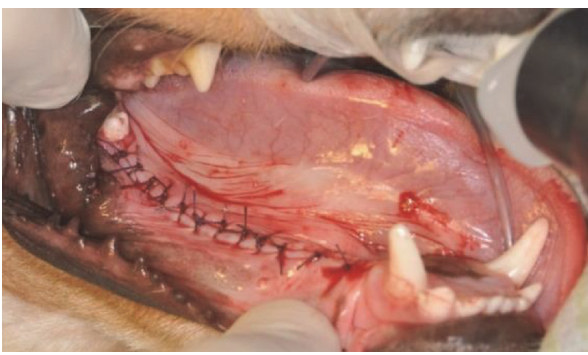

(d)

Figure 5: Clinical views of surgical procedure. (a) Forming the standardized defects. (b) Drilling for implant placement and forming the defects. (c) Implants placed with bone graft (Group 4, Group 2, Group 3, and Group 1). (d) Wound closure with suture.

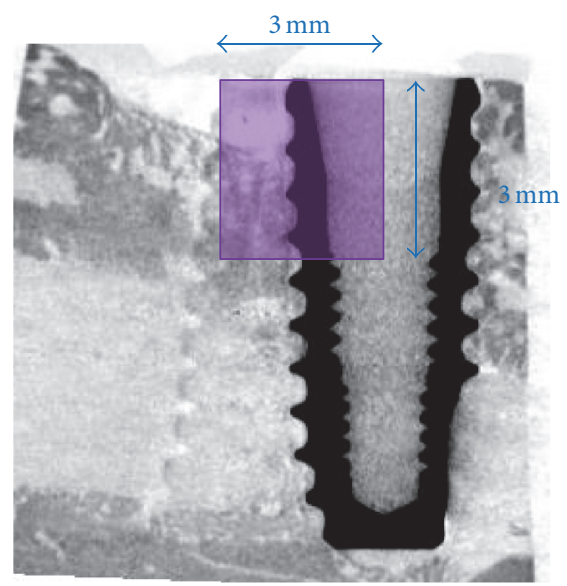

FIGURE 6: Bone volume measuring range in micro-CT image.

TABLE 2: The mean value of each ratio at 4 and 12 weeks.

\begin{tabular}{|c|c|c|c|c|c|c|c|c|}
\hline & \multicolumn{2}{|c|}{ Group 1} & \multicolumn{2}{|c|}{ Group 2} & \multicolumn{2}{|c|}{ Group 3} & \multicolumn{2}{|c|}{ Group 4} \\
\hline & 4 weeks & 12 weeks & 4 weeks & 12 weeks & 4 weeks & 12 weeks & 4 weeks & 12 weeks \\
\hline $\mathrm{BIC}$ ratio $(\%)$ & $42.73 \pm 31.27$ & $40.47 \pm 18.59$ & $13.59 \pm 19.22$ & $25.78 \pm 23.94$ & $47.98 \pm 32.96$ & $70.94 \pm 14.59$ & $28.11 \pm 19.11$ & $22.43 \pm 31.72$ \\
\hline $\begin{array}{l}\text { New bone area ratio } \\
(\%)\end{array}$ & $32 \pm 16.26$ & $48.53 \pm 13.61$ & $7.43 \pm 6.17$ & $14.2 \pm 15.74$ & $40.62 \pm 39.33$ & $55.49 \pm 29.57$ & $15.09 \pm 8.81$ & $5.28 \pm 3.2$ \\
\hline $\begin{array}{l}\text { Remaining graft } \\
\text { material area ratio } \\
(\%)\end{array}$ & & & $9.78 \pm 5.82$ & $12.08 \pm 9.57$ & & & $21.4 \pm 6.62$ & $16.48 \pm 6.31$ \\
\hline $\begin{array}{l}\text { Resorption area ratio } \\
(\%)\end{array}$ & $36.13 \pm 7.91$ & $46.09 \pm 17.99$ & $17.4 \pm 19.3$ & $39.23 \pm 35.91$ & $33.76 \pm 28.58$ & $12.15 \pm 17.19$ & $5.09 \pm 10.18$ & $21.79 \pm 17.66$ \\
\hline
\end{tabular}




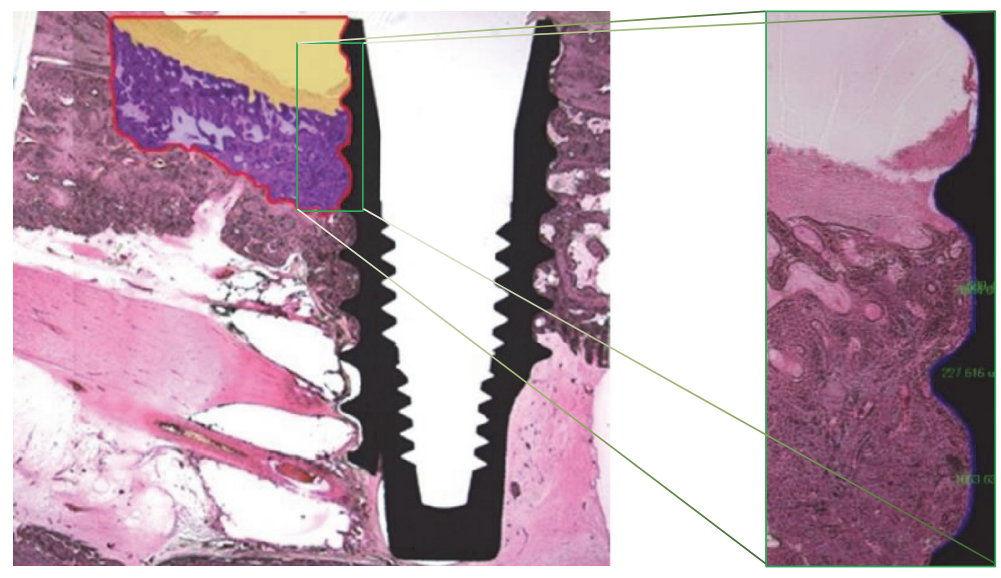

(a)

(b)

FIGURE 7: Histologic images showing experimental site, red box $\rightarrow$ ROI. (a) H\&E stained image showing experimental site ( $\times 12.5)$, purple zone $\rightarrow$ new bone area (\%) or remaining graft material area (\%), and yellow zone $\rightarrow$ resorption area (\%). (b) BIC in ROI, (H\&E stained, $\times 50.0$ ).

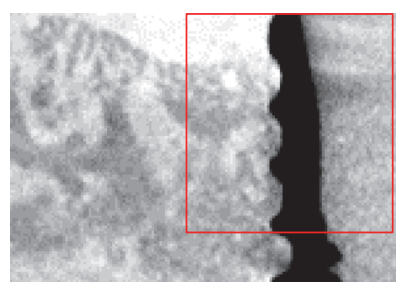

(a)

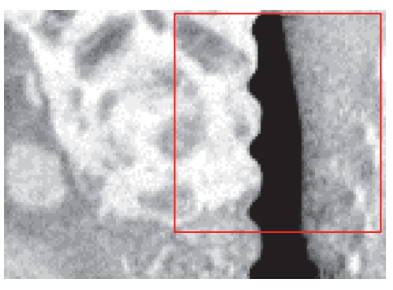

(b)

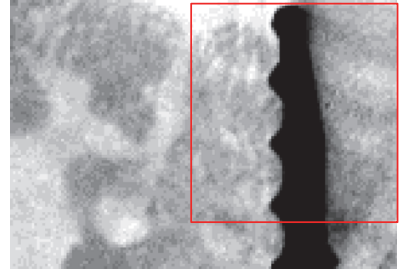

(c)

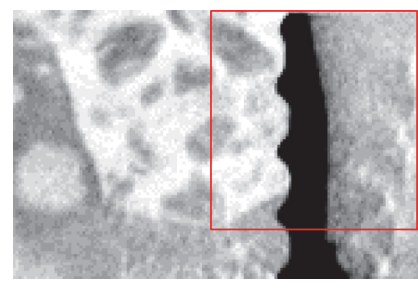

(d)

FIGURE 8: The representative micro-CT images of each group at 4 weeks. Red box $\rightarrow$ ROI in experimental site. (a) Group 1, (b) Group 2, (c) Group 3, and (d) Group 4.

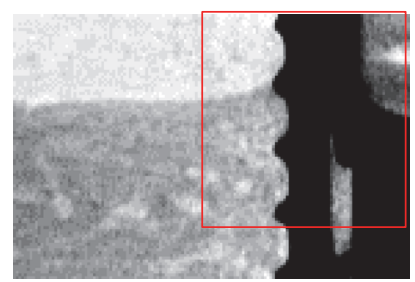

(a)

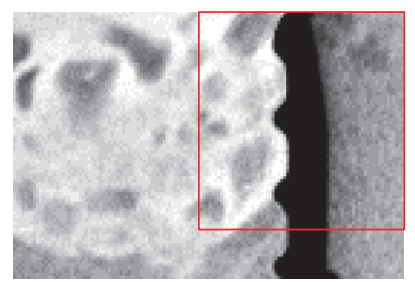

(b)

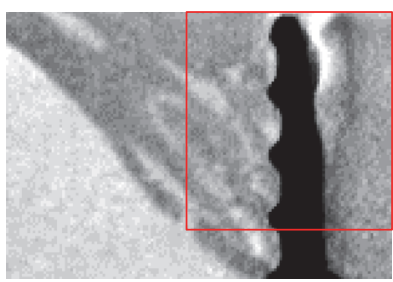

(c)

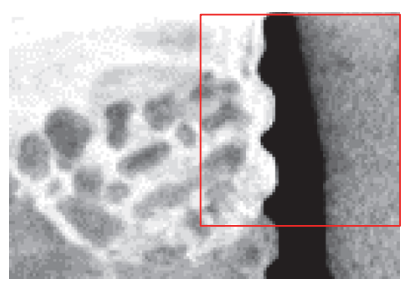

(d)

FIGURE 9: The representative micro-CT images of each group at 12 weeks. Red box $\rightarrow$ ROI in experimental site. (a) Group 1, (b) Group 2, (c) Group 3, and (d) Group 4.

in the group of implants placed over bone defect with graft, BIC ratios of UV-treated implant surfaces were greater than those at 4 weeks but lower than those at 12 weeks although there was no statistically significant difference in BIC ratio between UV-treated group and UV-untreated group. BIC values in Group 4 at 4 weeks were actually more than twice the BIC values in Group 2. On the other hand, BIC values in Group 4 at 12 weeks showed little difference from BIC values in Group 2. These results suggest that BIC of implant surfaces was increased by UV photofunctionalization for bone defects with a bone graft at the initial stage of the implant placement. The decrease in BIC values at 12 weeks could possibly be attributable to the characteristics of female beagle dogs, including concerns that they are more variable than males due to cyclical reproductive hormones [20], which may lead to a larger variation among the individuals. This can be related to the one of the limitations of the present study: relatively high standard deviation. A small number of sample sizes may also have resulted in such high standard deviation. To resolve these issues in further research and to further evaluate the effect of experimental intervention prior to the application in clinic, it may be necessary to use a larger number of male dogs.

Next, the new bone area ratio (\%) was measured in ROI. The mean of groups with UV photofunctionalization was slightly higher at both 4 and 12 weeks when the implant 


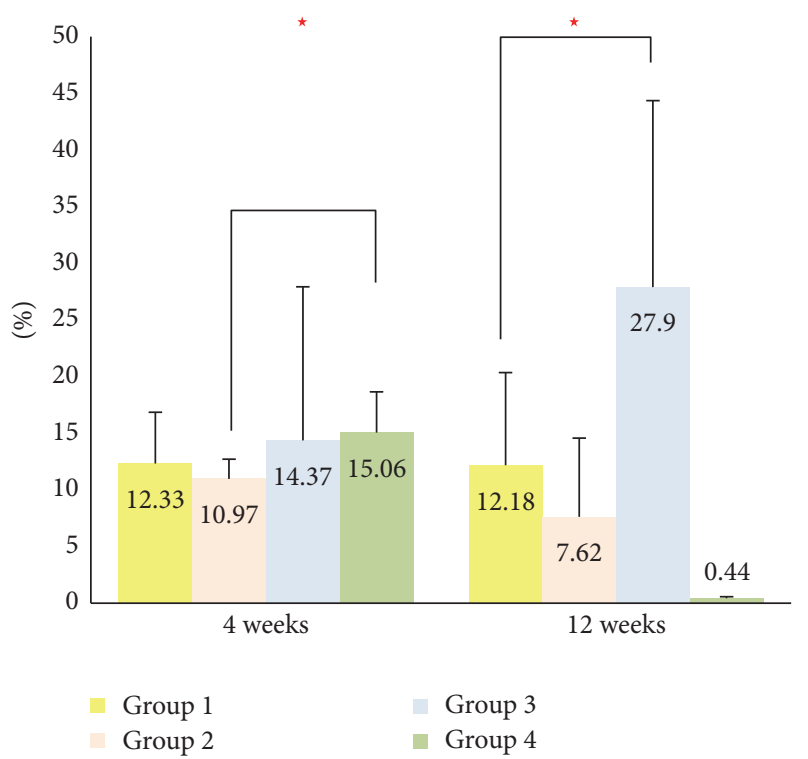

FIgURE 10: The mean of micro-CT value ratios at 4 and 12 weeks. Red star indicates that there was a significant difference $(p<0.05)$.

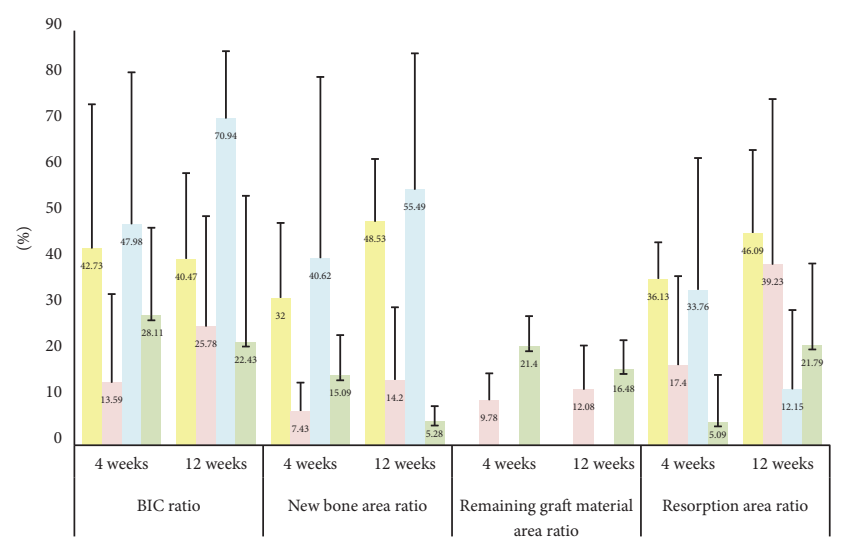

$$
\begin{array}{ll}
\text { Group } 1 & \text { Group } 3 \\
\text { Group } 2 & \text { Group } 4
\end{array}
$$

FIGURE 11: The mean value of each ratio at 4 and 12 weeks.

was placed without a bone graft, yet with no statistically significant difference. As UV photofunctionalization affected the bone defect area itself, more new bone might have been formed. Group 4 at 4 weeks had a greater new bone area ratio (\%) values than those of Group 2, which may indicate that the UV photofunctionalization had again a positive influence on early new bone formation for bone defect with a bone graft. However, Group 2 measurements at 12 weeks were higher than those in Group 4, which is in accordance with the results from the BIC ratio at 12 weeks and can be explained in the same extent.

The area of the remaining graft material ratio (\%) in ROI was measured for comparisons. The higher the remaining graft material ratio is, the more the graft material remained in the defect area and thus the greater the volume of the defect is maintained. Because the grafted, demineralized materials were absorbed rapidly, there were questions whether they could serve as a scaffold for a long time. In fact, Group 2 showed greater measurements at 4 weeks than at 12 weeks. Group 4 showed less remaining grafting material at 12 weeks. Based on comparisons of the actual measurements of each group, values were inconsistent and had large standard deviations at 12 weeks. In fact, it was previously reported that, in a one-wall defect, it is important to prevent dissipation of graft materials $[21,22]$. In the present study, however, no membrane or any other methods for maintenance of graft material were used and this may have resulted in inconsistent and large standard deviation at 12 weeks. Therefore, subsequent experiments may yield more reliable results if any methods for maintaining graft material are used.

The resorption area ratio (\%) reflected the degree of defect volume maintenance. In the group without bone graft, UV treatment showed little effect at 4 weeks. With time, the resorption area ratio decreased in Group 3. This maybe resulted from the large amount of new bone as well as from less resorption of bone itself. The differences in bone volume measured using micro-CT surely account for the differences in resorption area ratio. The amount of new bone was measured in three dimensions with a micro-CT scanner whereas the resorption area was measured in one plane with a light microscopy. These differences must be considered in calculating each measurement and may underlie limitations in calculating the resorption area using micro-CT measurements. For this reason, the implant placement position was considered the major cause of differences in resorption area ratio at 12 weeks. Further study should be required to employ more subdivided positions for implant placement in order to enable greater uniformity of placement. The resorption area ratio in Group 4 was less than in Group 2 at 4 weeks. This may be attributed to the greater amount of new bone and remaining graft material in Group 4 than in Group 2. Similarly, the resorption area ratio in Group 4 was less than in Group 2 at 12 weeks, a consequence of the difference in resorption rates due to posterior implant placement. Due to the difference in resorption rate caused by implant placement position, the amount of remaining graft material in Group 4 exceeded that of Group 2 and this may also explain the relatively reduced resorption area ratio in Group 4.

Regarding amount of new bone in ROI measured by micro-CT, in the group without bone graft, although the similar patterns in histomorphometric measurements at 4 weeks are identified, Group 3 measurements were about twice higher than those of Group 1 at 12 weeks. There was also statistically significant difference identified. These distinctions may be due to differences between measurement with light microscopy and micro-CT scanner as mentioned above. In the bone graft groups, Group 4 scored higher in new bone volume measurements than Group 2 at an early stage ( 4 weeks). This agrees with the pattern of new bone formation measurements using a light microscopy.

Within the limitation of the present study, based on aforementioned results, it can be concluded that UV photofunctionalization had a positive effect on new bone formation of bone defect with bone graft in the early stage. However, the 
results of each group in a late stage ( 12 weeks) were not significantly correlated with the presence of UV treatment and this may be partly attributed to the fact that the results of these experiments do not completely rule out the effect of implant location. Therefore, further study will be required to maintain uniform implant conditions including placement location as much as possible by increasing the number of sample sizes and by subdividing the location of implant placement.

\section{Disclosure}

Young-Bum Park is co-corresponding author for supervising overall animal study. This paper is a part of Min-Young Kim's thesis.

\section{Competing Interests}

The authors declare that they have no competing interests.

\section{Authors' Contributions}

Min-Young Kim and Hyunmin Choi equally contributed to the works described in this manuscript.

\section{Acknowledgments}

This research was supported by a faculty research grant of Yonsei University College of Dentistry (6-2015-0106), Basic Science Research Program, through the National Research Foundation of Korea (NRF) funded by the Ministry of Education (NRF-2016R1D1A1B03934584) and Institute for Information \& Communications Technology Promotion (IITP) grant funded by the Korea Government (MSIP) (no. R019015-1113).

\section{References}

[1] T. Ogawa, "Ultraviolet photofunctionalization of titanium implants," The International journal of oral \& maxillofacial implants, vol. 29, no. 1, pp. e95-102, 2014.

[2] T. Berglundh, I. Abrahamsson, N. P. Lang, and J. Lindhe, "De novo alveolar bone formation adjacent to endosseous implants: a model study in the dog," Clinical Oral Implants Research, vol. 14 , no. 3, pp. 251-262, 2003.

[3] J. E. Davies, "Mechanisms of endosseous integration," International Journal of Prosthodontics, vol. 11, no. 5, pp. 391-401, 1998.

[4] H. Aita, N. Hori, M. Takeuchi et al., "The effect of ultraviolet functionalization of titanium on integration with bone," Biomaterials, vol. 30, no. 6, pp. 1015-1025, 2009.

[5] A. Funato and T. Ogawa, "Photofunctionalized dental implants: a case series in compromised bone," The International journal of oral \& maxillofacial implants, vol. 28, no. 6, pp. 1589-1601, 2013.

[6] A. Funato, M. Yamada, and T. Ogawa, "Success rate, healing time, and implant stability of photofunctionalized dental implants," The International Journal of Oral \& Maxillofacial Implants, vol. 28, no. 5, pp. 1261-1271, 2013.

[7] D. Buser, V. Chappuis, M. M. Bornstein, J.-G. Wittneben, M. Frei, and U. C. Belser, "Long-term stability of contour augmentation with early implant placement following single tooth extraction in the esthetic zone: a prospective, cross-sectional study in 41 patients with a 5- to 9-year follow-up," Journal of Periodontology, vol. 84, no. 11, pp. 1517-1527, 2013.

[8] G. E. Friedlaender, "Bone grafts. The basic science rationale for clinical applications," The Journal of Bone \& Joint SurgeryAmerican Volume, vol. 69, no. 5, pp. 786-790, 1987.

[9] R. E. Jung, N. Fenner, C. H. F. Hämmerle, and N. U. Zitzmann, "Long-term outcome of implants placed with guided bone regeneration (GBR) using resorbable and non-resorbable membranes after 12-14 years," Clinical Oral Implants Research, vol. 24, no. 10, pp. 1065-1073, 2013.

[10] A. Stavropoulos, L. Kostopoulos, J. R. Nyengaard, and T. Karring, "Deproteinized bovine bone (Bio-Oss) and bioactive glass (Biogran) arrest bone formation when used as an adjunct to guided tissue regeneration (GTR): an experimental study in the rat," Journal of Clinical Periodontology, vol. 30, no. 7, pp. 636643, 2003.

[11] D. Carmagnola, T. Berglundh, M. Araújo, T. Albrektsson, and J. Lindhe, "Bone healing around implants placed in a jaw defect augmented with Bio-Oss. An experimental study in dogs," Journal of Clinical Periodontology, vol. 27, no. 11, pp. 799-805, 2000.

[12] F. A. Santos, M. T. Pochapski, M. C. Martins, E. G. Zenóbio, L. C. Spolidoro, and E. Marcantonio Jr, "Comparison of biomaterial implants in the dental socket: histological analysis in dogs," Clinical Implant Dentistry and Related Research, vol. 12, no. 1, pp. 18-25, 2010.

[13] M. Marco Degidi, A. Piattelli, V. Perrotti, and G. Iezzi, "Histologic and histomorphometric evaluation of an implant retrieved 8 years after insertion in a sinus augmented with anorganic bovine bone and anorganic bovine matrix associated with a cell binding peptide," The International Journal of Periodontics \& Restorative Dentistry, vol. 32, pp. 451-457, 2012.

[14] A. Philipp, W. Duncan, M. Roos, C. H. Hämmerle, T. Attin, and P. R. Schmidlin, "Comparison of SLA ${ }^{\circledR}$ or SLActive ${ }^{\circledR}$ implants placed in the maxillary sinus with or without synthetic bone graft materials-an animal study in sheep," Clinical Oral Implants Research, vol. 25, no. 10, pp. 1142-1148, 2014.

[15] T. Ueno, M. Yamada, N. Hori, T. Suzuki, and T. Ogawa, "Effect of ultraviolet photoactivation of titanium on osseointegration in a rat model," The International Journal of Oral \& Maxillofacial Implants, vol. 25, no. 2, pp. 287-294, 2010.

[16] T. Ueno, M. Yamada, T. Suzuki et al., "Enhancement of bonetitanium integration profile with UV-photofunctionalized titanium in a gap healing model," Biomaterials, vol. 31, no. 7, pp. 1546-1557, 2010.

[17] J. C. de Vicente, O. Recio, L. Martín-Villa, L. M. Junquera, and J. S. López-Arranz, "Histomorphometric evaluation of guided bone regeneration around implants with SLA surface: an experimental study in beagle dogs," International Journal of Oral and Maxillofacial Surgery, vol. 35, no. 11, pp. 1047-1053, 2006.

[18] K. Donath, "Equipment and methodical performance," in Preparation of Histologic Sections, EXAKT-Kulzer-Publication, Norderstedt, Germany, 1995.

[19] A. K. Gosain, L. Song, P. Yu et al., "Osteogenesis in cranial defects: reassessment of the concept of critical size and the expression of TGF- $\beta$ isoforms," Plastic and Reconstructive Surgery, vol. 106, no. 2, pp. 360-371, 2000.

[20] I. Zucker and A. K. Beery, "Males still dominate animal studies," Nature, vol. 465, no. 7299, p. 690, 2010.

[21] J. Sakata, H. Abe, A. Ohazama et al., "Effects of combined treatment with porous bovine inorganic bone grafts and bilayer 
porcine collagen membrane on refractory one-wall intrabony defects," International Journal of Periodontics and Restorative Dentistry, vol. 26, no. 2, pp. 161-169, 2006.

[22] J.-I. Park, C. Yang, Y.-T. Kim et al., "Space maintenance using crosslinked collagenated porcine bone grafted without a barrier membrane in one-wall intrabony defects," Journal of Biomedical Materials Research B Applied Biomaterials, vol. 102, no. 7, pp. 1454-1461, 2014. 


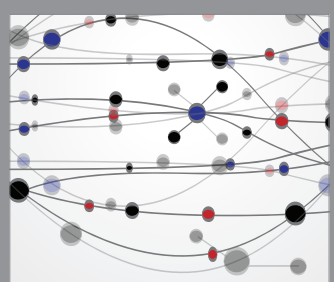

The Scientific World Journal
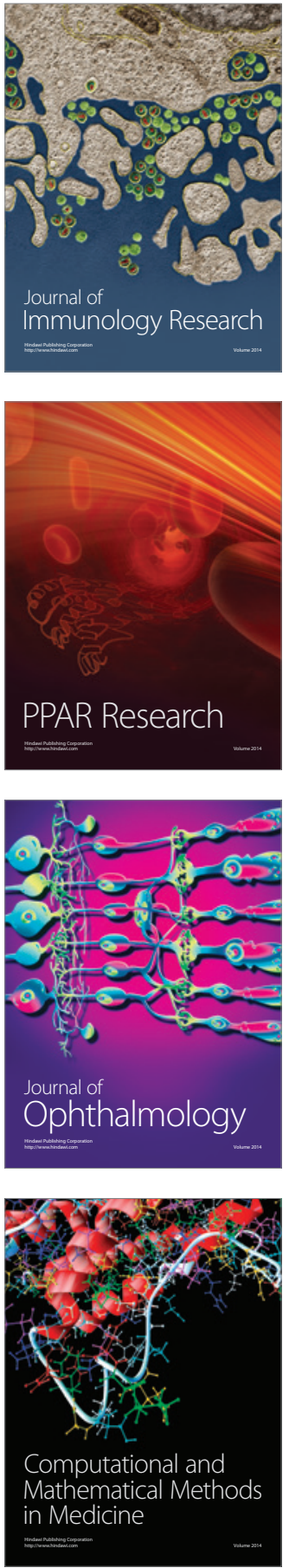

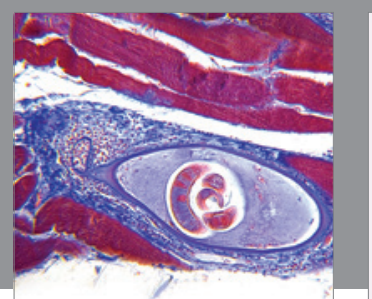

Gastroenterology Research and Practice

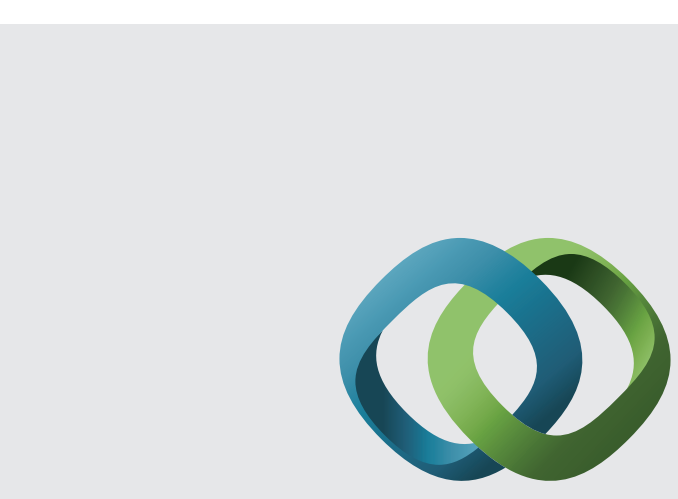

\section{Hindawi}

Submit your manuscripts at

http://www.hindawi.com
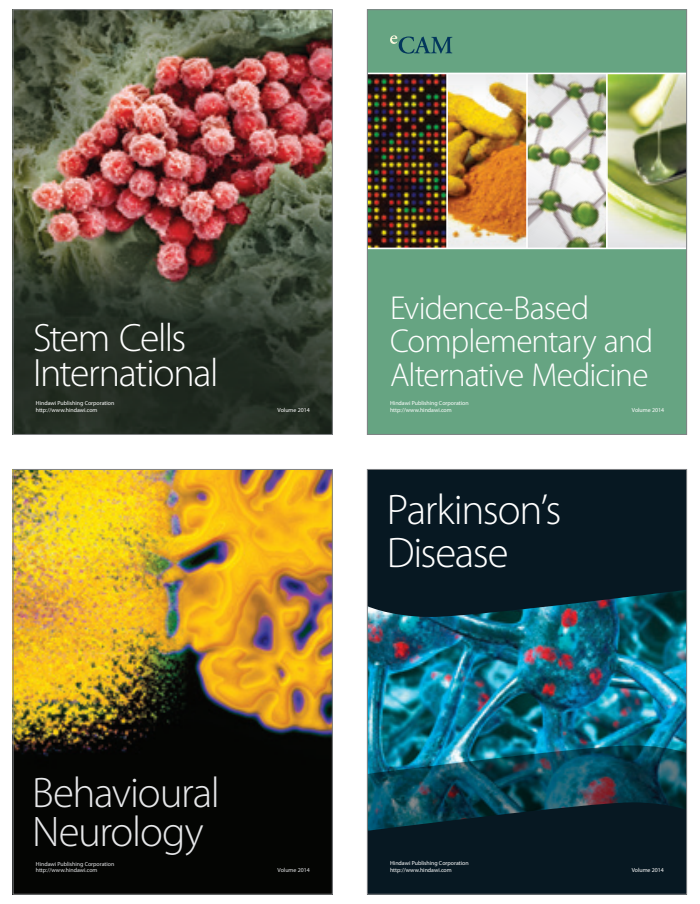
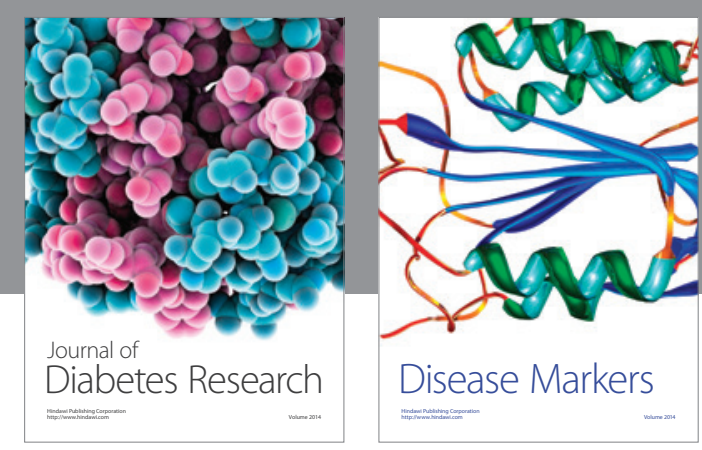

Disease Markers
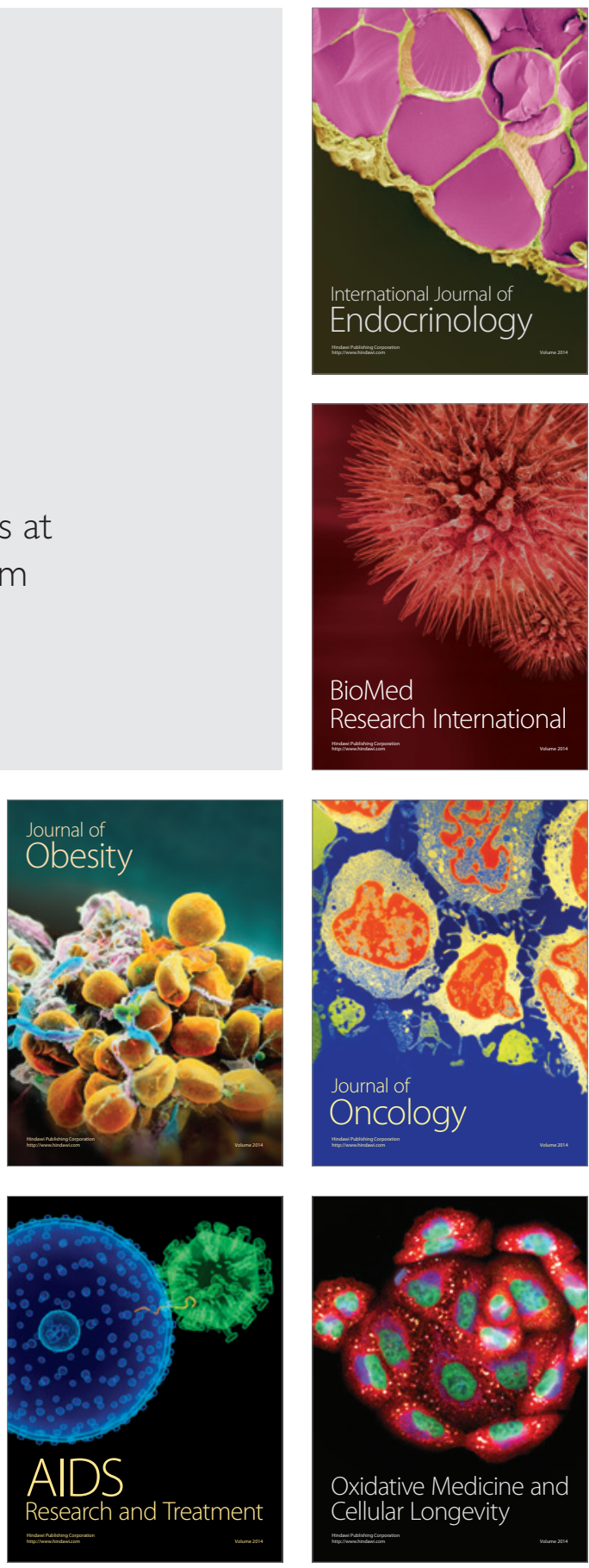